\title{
Use of Prefabrication, Construction and Demolition Wastes as an Aggregate in Vibropressed Precast Concrete Blocks Production
}

\author{
Grzegorz Łój, Wiesława Nocuń-Wczelik* \\ AGH - University of Science and Technology, al.Mickiewicza 30, 30-059Kraków, Poland \\ E-mail: wiesia@agh.edu.pl (Corresponding author)
}

Received: 31 May 2021; Accepted: 4 November 2021; Available online: 25 November 2021

\begin{abstract}
The aim of current study was to determine the recycled concrete aggregate (RCA) applicability in the production of concrete mixture for vibropressed concrete blocks. The experiments were focused especially on the crushed waste material from the same concrete elements producing plant. For this type of precast elements only some finer fractions can be implemented and the "earth-moist" consistency of fresh mixture is required. The series of samples was prepared in which the mixture of natural aggregates was partially or totally substituted by recycled concrete aggregate. The 0/4 RCA fraction, which is usually rejected in ready mix concrete technology, plays a role of $0 / 2$ sand. The substitution of sand fraction was from $20 \%$ to $100 \%$ respectively. The substitution of the coarser aggregate fractions by 4/16 RCA was also done. The standard properties of vibropressed elements, such as the degree of densification, the density of material, the compressive and splitting tensile strength and the water absorption capacity according to the relevant standards were determined. The parameters of materials with the natural aggregate substitution by RCA are affected by the ratio of recycled concrete aggregate. In most cases the results do not decline specially from those for reference samples, when only the natural sand $(0 / 2)$ fraction is substituted by the $0 / 4$ recycled aggregate. As one could expect, as lower the substitution, as better the test results. The partial substitution of natural aggregate by coarser fractions requires experimental verification; over $20 \%$ substitution of natural aggregate by 4/8, 8/16 or 0/16 RCA should be excluded.
\end{abstract}

Keywords: Recycled concrete aggregate; Vibropressed concrete; Compacting; Density; Strength; Absorbability.

\section{Introduction}

The production of concrete small vibropressed elements has been bound first of all to the development of modern urban road infrastructure. The state-of-the-art and the potential of the interlocking concrete block pavement was thoroughly discussed based on the laboratory and field experience by Japanese authors in a comprehensive review paper [1]. The sustainability and social background were taken into account as well. The relevant literature in English and Japanese, including journals, conference proceedings, technical reports, books, and theses, over a span of 47 years (1971-2018), were studied and over 300 references are cited. The authors come to the conclusion that owing to the waste materials use in the form of supplementary cementing materials, less noise emission, air purifying characteristics and heat emission reduction, the environmental performance of interlocking concrete block pavement are in harmony with sustainable practices.

In this study the use of recycled concrete aggregate in the production of concrete paving blocks was investigated. The RCA is successfully used in the production of ready mix concrete for construction but there is almost no reports dealing with the RCA implementation in the prefabrication of small, prefabricated elements. In the experiments the "in situ" wastes from the concrete blocks producing plants were taken into account with the same other materials, such as cement, aggregate and admixtures used in current production. One should underline that this RCA is not contaminated by chlorides and sulfates (e.g. from de-icing salts or seawater). Thus the problem of waste (e.g. damaged products) management in any concrete elements producing plant could be resolved. At the same time some amount of natural aggregate could be replaced by the recycled material. Only a few studies can be found in the literature that deal with the improvement of concrete paving blocks made with recycled aggregate. However, the authors can relate to the numerous reports dealing with the re-use of RCA in ready mix concrete production which are relatively abundant.

One should take in mind that in concrete mixtures used to produce the vibropressed non-construction concrete materials the recycled aggregate fraction $0 / 4$, which is usually rejected in ready mix concrete technology, can successfully replace the $0 / 2$ natural sand fraction. This is of importance because of the preservation of natural sand used as a raw material in many building materials production. 
In the next chapters the question of composition of concrete mixture used in the vibropressed precast concrete technology is highlighted. Subsequently, the general problem of recycled concrete aggregate is discussed with some aspects of RCA use in paving blocks production. Finally the experimental part and the conclusions are presented.

\section{The production of concrete mixture used in the vibropressed precast concrete technology}

\subsection{The components of concrete mixture}

The following components are used in the production of concrete mixture for the vibropressed precast concrete paving blocks: Portland cement, fine aggregate, mineral additives and chemical admixtures. The fly ash from power plants is the most commonly used mineral additive in this case; the blended fly ash or slag Portland cement as a component of concrete mixture can be used as well [2]. The fresh concrete mixture must be very rigid, more compact than the material of wet consistency (Ve-Be > 31s). Simultaneously, the mixture should be produced at low water to cement ratio, on the level $0.34-0.38$. Therefore some additional technological actions should be undertaken in order to warrant a fairly good workability. Among them a proper grain size distribution of aggregate is critical, as well as the use of chemical admixtures. The optimization of fine grained dust component (fraction $\leq$ $0,125 \mathrm{~mm}$ ) is necessary. This fine-grained component consists of the finest fraction of all the components: aggregate, cement and mineral additives. These factors should be taken into account as the requirements in respect to the recycled aggregates are formulated. The optimum fine-grained component content, to ensure the proper vibropressing operation of concrete mixture is estimated generally for $320-380 \mathrm{~kg} / \mathrm{m}^{3}$ of concrete. These values can be altered; it depends upon the particular properties of materials, technology, etc. However, the best compaction of elements and, as a consequence, the best parameters of product are the main goals.

The wet concrete mixture can be discussed as a rheological object, on the basis of ground mechanics, taking into account the rules governing the adhesion and friction forces. These forces act between the grains of different shape and surface roughness. The cement grains in the mixture can be then considered as a fine, at first stage inactive chemically aggregate fraction, affecting the water demand and susceptibility to densification. This latter feature is, beside of the early strength, a crucial factor indicating the applicability of the mixture. The consistency in this case cannot be measured with help of standard methods attributed to the typical concrete mixtures and cannot be classified according to the standard classification [3].

\subsection{The use of recycled aggregate in concrete - general aspects}

There is a common opinion that using recycled aggregates from construction and demolition waste can preserve natural aggregate resources, reduce demand of landfill, and contribute to sustainable built environment. There is a significant number of reports on the problem of construction and demolition wastes, their re-use as recycled concrete aggregate after crushing, milling, classification and separation of contaminants [4-11]. Some of the stateof-the art papers appeared recently [12-14]. The effect of parent concrete properties on the quality of concrete aggregate was studied in many research centers [9]. As it has been found for many years studies and practical trials, the recycled aggregate can be successfully used in the production of ready mix and precast concrete, as a partial or whole substitute of natural aggregate, particularly since the amount of construction and demolition wastes continuously grows. In practice, each concrete waste can be subjected to the recycling aimed in the production of valuable "secondary" aggregate.

Owing to its adhered cement paste, which is of porous nature, RCA implementation should be expected to affect concrete performance at all stages of concrete production and use (fresh mixture, engineering properties, durability). The other key limiting property of RCA is identified as the high water absorption, which may lower concrete workability. The methods aimed in enhancing the RCA role as mechanical properties and long-term performance improving agent are summarized and categorized into three groups, i.e. the reduction of recycled aggregate porosity, reduction of old mortar layer on recycled aggregate surface, and the property improvement without recycled aggregate modification (appropriate concrete mixing design, addition of fiber reinforcement).

According to Rodriguez et al. [10] a 20\% replacement of coarse aggregate will cause no loss of properties, however the recycle aggregate can be used at greater rates for non-structural uses. The manufacturing of paving blocks is an example [10, 15-17].

The wastes during the paving block and other precast elements manufacturing are generated too and they should be used "in situ”. However, there are some detailed questions dealing with the implementation of these wastes in vibropressed concrete technology. The concrete mixture is manufactured as an earth-moist, packed material. Finally, the ready elements must comply with relevant standard requirements in respect for example to the absorption capacity, strength and generally the durability. 


\subsection{Recycled aggregate - classification and requirements}

The three categories of aggregates are given in the EN 12620: 2002+A1:2008 standard "Aggregates for concrete" [18]; among them there are the recycled ones. However, there is no detailed guidance dealing with their application. In the appendix expired in 2008 there is a classification of recycled aggregate based on the ratio of uncombined stone material $(\mathrm{Ru})$, concrete waste $(\mathrm{Rc})$, waste from wall elements $(\mathrm{Rb})$, bituminous materials $(\mathrm{Ra})$, glass (Rg), unstable material (FL) or the other components.

The three types of recycled aggregate are indicated by RILEM:

1) Type I - waste from wall elements,

2) Type II - waste from concrete elements,

3) Type III - blended material composed at least from $80 \%$ natural aggregate and $20 \%$ recycled aggregate.

The recycled concrete aggregate (RCA) covers three classes, according to their potential use:

1) Class A - recycled aggregate for concrete of general purpose, including see and environmental structures;

2) Class B - mixed natural and recycled aggregate to be used in the production of concrete exposed to the environment of moderate aggressiveness;

3) Class C - recycled aggregate to be used in the production of concrete exposed to the environment of low aggressiveness [4].

However, the lack of the other standards for quality evaluation is the main reason for not limited using RCA in some concrete technologies outside the laboratory.

\section{Experimental}

The aim of current study was to determine the recycled aggregate applicability in the production of concrete mixture for vibropressed concrete blocks, based upon the results of relevant experiments. The mixture in this technology differs significantly from that for precast concrete products because of the specific aggregate granulation. Only some finer fractions can be implemented in this type of elements. The "earth-moist" consistency which is not used in the case of ready mix concrete is achieved by vibropressing compaction method.

In order to better simulate the conditions of vibropressed concrete paving blocks manufacturing the laboratory paving block machine, so-called Econom compaction apparatus was put into use [2,3]. In this experimental procedure the material is packed and thus compacted. The laboratory vibropress is used, accompanying the specimens shaping by impacts and pressing and the process of technical scale concrete elements production is thus well simulated.

\subsection{Materials}

The three samples of crushed concrete paving blocks from the vibropressed concrete paving blocks producing plant were taken as recycled aggregate:

1) The recycled concrete aggregate (RCA) 0/16 obtained by simple one-step crushing and sieving,

2) The recycled concrete aggregate (RCA) fraction $4 / 16$,

3) The recycled concrete aggregate (RCA) fraction $0 / 4$,

The aggregate fractions are presented in fig. 1 .

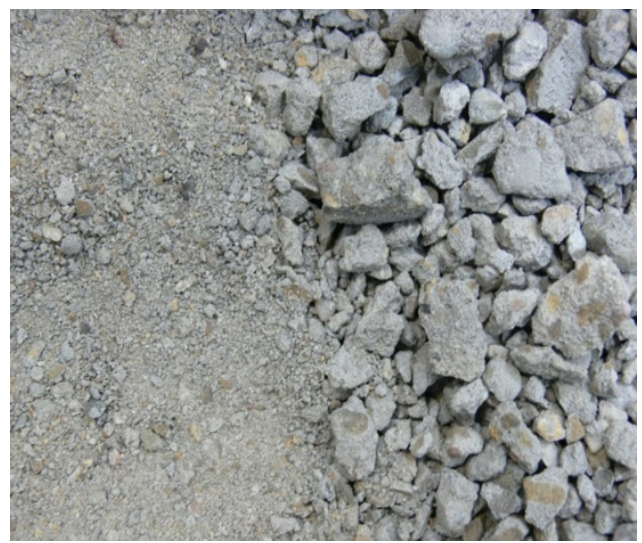

Fig. 1. Recycled concrete aggregate fractions $0 / 4$ (left side) and 0/16 (right side).

The typical aggregates used in the vibropressed concrete paving blocks technology were used as reference and as supplementary aggregate materials: 
1) Washed sand fraction $0 / 2$,

2) Washed gravel fraction $2 / 8$,

3) Washed gravel fraction $8 / 16$.

As one can see above, the grain size classification in the practice of concrete mixture production for vibropressing is different from that proposed by RILEM for recycled concrete aggregates. The finest RCA 0/4 fraction is "boarder" that the $0 / 2$ sand fraction.

The physical and chemical properties of natural and recycled aggregates were examined, apart from the standard sieve analysis; the results are shown in table 1.

The dust component ratio, it means the percentage of fraction below $0,063 \mathrm{~mm}$, is in the recycled concrete aggregate always much higher than in natural aggregate used in the vibro-pressed concrete technology. This is clearly seen particularly in the case of recycled aggregate fraction $0 / 4$ in which the dust component on the level $7,8 \%$ is almost 40 times higher than in the natural washed sand. Therefore an increase of water demand of aggregate and subsequently the lower workability is expected.

Table 1. The properties of natural and recycled aggregates

\begin{tabular}{|c|c|c|c|c|c|c|c|}
\hline \multirow{2}{*}{ Parameter } & & \multicolumn{4}{|c|}{ Natural aggregate } & \multicolumn{2}{|l|}{$\begin{array}{l}\text { Recycled } \\
\text { aggregate }\end{array}$} \\
\hline & & Sand & $0 / 2$ & $\begin{array}{l}\text { Gravel } \\
2 / 8\end{array}$ & $\begin{array}{l}\text { Gravel } \\
8 / 16 \\
\end{array}$ & Fraction $0 / 4$ & $\begin{array}{l}\text { Fraction } \\
4 / 16\end{array}$ \\
\hline Volume density $\rho_{a}$ & $\mathrm{~g} / \mathrm{cm}^{3}$ & 2,67 & & 2,63 & 2,63 & 2,47 & 2,53 \\
\hline Absorbability $\mathrm{WA}_{24}{ }^{*}$ & $\%$ & 0,33 & & 1,43 & 1,61 & 4,18 & 4,71 \\
\hline Freeze-thaw resistance $\mathrm{M}_{25}$ & $\%$ & - & & $0,85 \pm 0,24$ & $0,36 \pm 0,04$ & - & $1,56 \pm 0,14$ \\
\hline Flatness index FI ${ }^{*}$ & $\%$ & - & & 5 & 14 & - & 6 \\
\hline
\end{tabular}

However, in the case of concrete mixtures of wet, compact consistency, a negative impact of the higher content of dust fraction on the workability can be reduced markedly because the vibropressing is used as a compacting technique. The chemical admixtures can be applied too.

The list of concrete mixtures with the notation attributed to the type of aggregates used is as follows:

1) R0 - reference concrete mixture, $w / c=0,36$; the composition of this mixture is given in table 2; because of the variable w/c ratio in case of the mixtures with RCA substitution the R01 reference samples with low w/c=0,27 was also produced (sample no1);

2) R20, R50 and R100 - series of concrete mixtures in which the mixture of natural aggregates was substituted by a recycled, not fractioned 0/16 concrete aggregate (RCA). The substitution was 20\%, 50\% and 100\% respectively (samples no 2, 3, 4);

3) Rc25, Rc50 and Rc100 - series of concrete mixtures in which the mixture of 2/8 and 8/16 gravels was substituted by $4 / 16$ RCA. The substitution was $25 \%, 50 \%$ and $100 \%$ respectively (samples no $5,6,7$ );

4) Rf25, Rf50, Rf100 - series of concrete mixtures in which only the washed sand $0 / 2$ was substituted by a recycled concrete aggregate (RCA) fraction $0 / 4 \mathrm{~mm}$. The sand substitution was $25 \%, 50 \%$ and $100 \%$ respectively (samples no 8, 9,10).

Table 2. The composition of reference concrete mixture.

\begin{tabular}{ll}
\hline Component & Content, $\left[\mathbf{k g} / \mathbf{m}^{3}\right]$ \\
\hline Cement CEM II/A-V 42,5 R & 340 \\
Sand 0/2 & 714 \\
Gravel 2/8 + 8/16 (1:1 ratio) & 1190 \\
Water (initially, before correction) & 122,4 \\
Plastifier & $0,46 \%$ by mass of cement \\
w/c & $0,36(0,27)$ \\
\hline
\end{tabular}

The natural aggregate was substituted in the mixtures according to the scheme presented above. The mineral additive was not used but instead of the CEM I type cement, the fly ash Portland blended cement CEM II/A-V was added. 


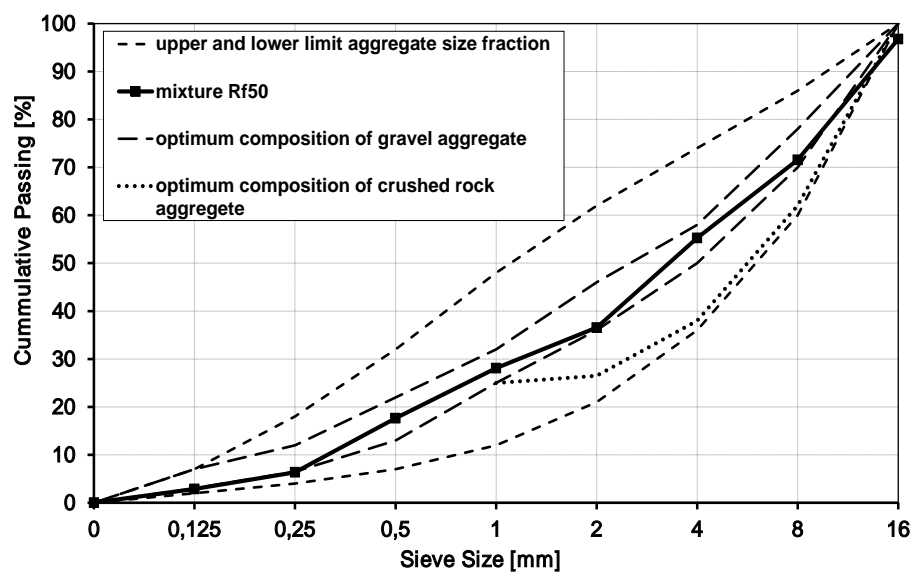

Fig. 2. Grain size cumulative curves for aggregate in which the $0 / 4$ RCA substitutes for $0 / 2$ natural sand (Rf50 aggregate mixture).

It was found that the grain size cumulative curves were within the limits recommended for the aggregate to concrete. As an example, the grain size distribution curve for the recycled concrete aggregate material $0 / 4$ substituting the $0 / 2$ sand fraction is shown in fig. 2 .

Several concrete mixtures were produced based on different aggregates assemblages listed above (R0, R01, R20, R50, R100, Rc25, Rc50, Rc100, Rf25, Rf50, Rf100). Their consistency/workability was adequate to the vibropressing technology. The amount of water used for every dosage was modified to obtain the earth-moist consistency and consequently the fairly well shaped elements.

\subsection{Methods of concrete treatment and examination}

Concrete specimens were prepared in the so-called Econom compactor used in the experimental verification of concrete mixture design for vibropressed small elements; the mixture is vibrated during filling the vessel with particular components, starting from the aggregate [2].

The degree of densification; it means the calculated density of material filling the mold to the density of material after vibropressing ratio, was measured in Econom device, in which the vibropressing action was simulated.

The compressive and splitting tensile strength of pavement blocks were determined by tests at 7- and 28-days curing, according to the EN 1338 [19] and EN 15037-2 [20] standards respectively. The specimens were cured in the conditions recommended by the EN 12390-7:2011 [21] standard (in water, at temperature $20 \pm 5^{\circ} \mathrm{C}$ ).

Water absorption tests of concretes were carried out on the pavement blocks matured for 28 days, according to the EN 1338 standard.

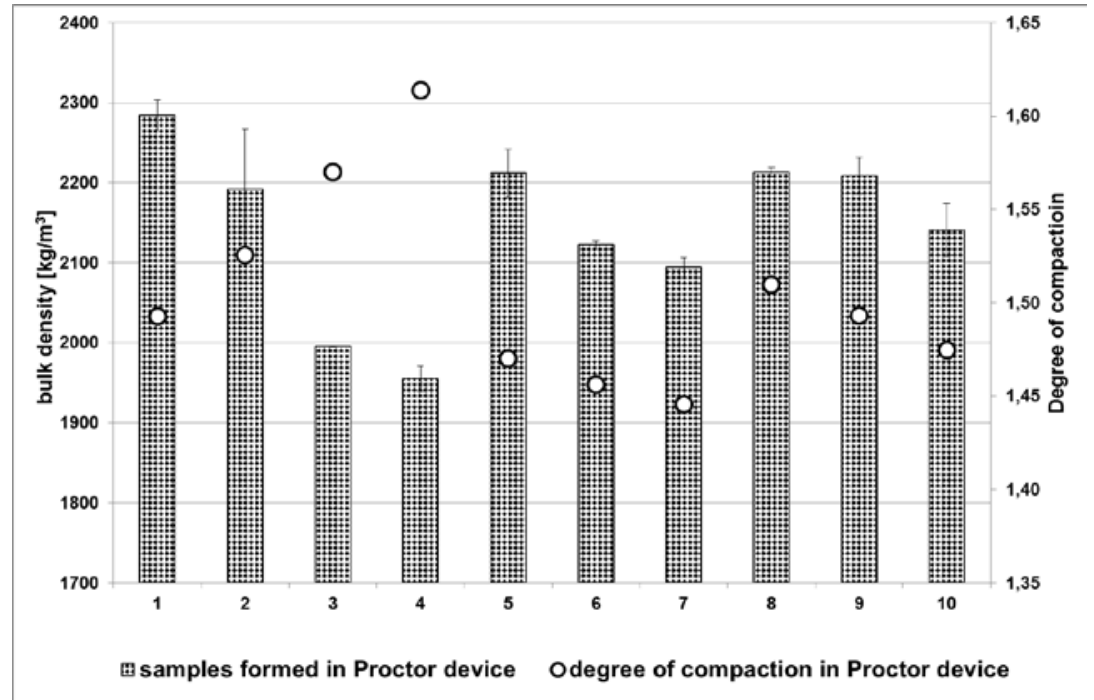

Fig. 3. Density and degree of compaction of concrete samples 


\subsubsection{Density of concrete samples}

The density of concrete samples was determined according to the EN 12390-7:2011 standard after 28-day maturing in water at temperature $20 \pm 5^{\circ} \mathrm{C}$ [21]. This method, based on the measurements of water dislodgement gives the possibility to evaluate the density of concrete elements formed with help of different methods. The results, shown in fig 3. are the average values from several individual measurements of samples produced using Econom apparatus.

The density of hardened concretes is affected by the type and amount of recycled concrete aggregate. In the concrete with RCA the decrease of density with an increase of recycled concrete ratio is observed. The reduction of density is the consequence of lower RCA density resulting, in turn, from the porosity of residual hydrated hardened cement material being the constituent of RCA. Only the Rf50 concrete with substitution of $50 \%$ sand by RCA 0/4 mm shows a slight increase of density in respect to the Rf25 material. This is the consequence of better concrete mixture compactness in the case of Rf50 than Rf25.

The most significant reduction of density, as compared to the value for reference R0, is found for concretes with $0 / 16$ recycled concrete aggregate; the difference in case of R100 is close to $15 \%$. The sample of concrete R01 with reduced water content $(\mathrm{w} / \mathrm{c}=0,27)$ obviously shows a lower density than the material with w/c=0,36. At lower water content there is no sufficient amount of paste to have fairly good compacting during the formation of blocks. The mixture R01 appeared less compact at loose state than the R0 material.

The testing with help of Econom device gives the possibility to investigate the densification process as a function of time. This is illustrated in fig. 4. The susceptibility to densification was determined during shaping and vibropressing of concrete blocks from concrete mixtures R0, R100 (with 100\% 0/16 RCA), Rc100 (RCA 4/16 substitution for 2/8 + 9/16 gravel fraction) and Rf100 (RCA 0/4 instead of 0/2 natural sand) respectively.

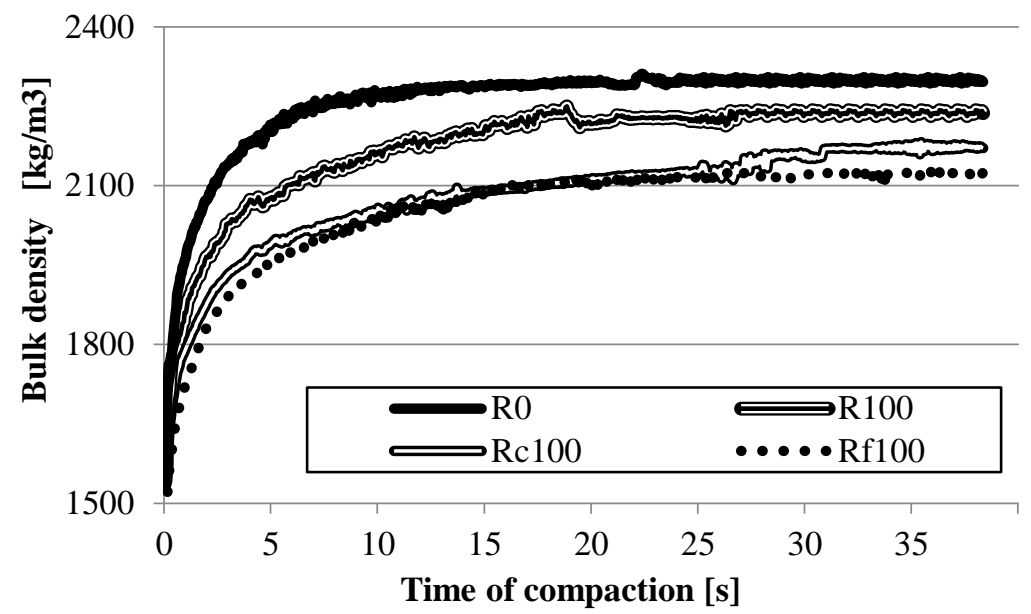

Fig 4. The density of concrete mixtures vs. time of vibropressing

Generally, as one can notice in fig. 4, the recycled concrete aggregate brings about some acceleration of densification operation, presumably because of an excess of finest fractions filling the spaces between the coarser aggregate grains.

\subsubsection{Compressive and splitting tensile strength determination}

The splitting tensile strength of concrete was determined according to the EN 12390-6:2011 standard [21], the compressive strength was examined according to the EN 12390-3:2011 standard on the cylindrical samples after 7- and 28- day maturing. The samples were formed and subsequently cured in the conditions recommended by the EN 12390-7:2011 [21] standard (in water, at temperature $20 \pm 5^{\circ} \mathrm{C}$ ). The surface and fractured cylindrical samples morphology is shown in fig. 5. The results of compressive and splitting tensile strength of concrete after 7- and 28-day maturing are shown in table 3.

The results in respect to the compressive and splitting tensile strength of concrete blocks can be summarized as follows:

1) The mechanical properties of vibropressed concrete elements produced with recycled concrete aggregate are strongly affected by the aggregate grain size and aggregate ratio. Obviously, as lower the substitution, as better the test results. Only the samples produced Rf25, Rf50, Rf100 concrete mixtures, in which the RCA 0/4 was used instead of 0/2 natural sand meet the requirements of EN 1338:2005+AC2007 [19] standard in respect to the minimum splitting tensile strength for paving block elements - not lower than 3,6MPa. It means that the 0/4 RCA fraction introduced instead of the 0/2 natural sand improves the strength of vibro-pressed concrete. These results 
are in good agreement with the data of Husken and Brouwers in respect to the mix design concept optimization for earth-moist concrete [22]. The samples produced from R20 (20\% 0/16 RCA), Rc25 (25\% RCA substitution for $2 / 8+9 / 16$ gravel fraction) meet these requirements too. These data are in good agreement with the ones previously reported [10].

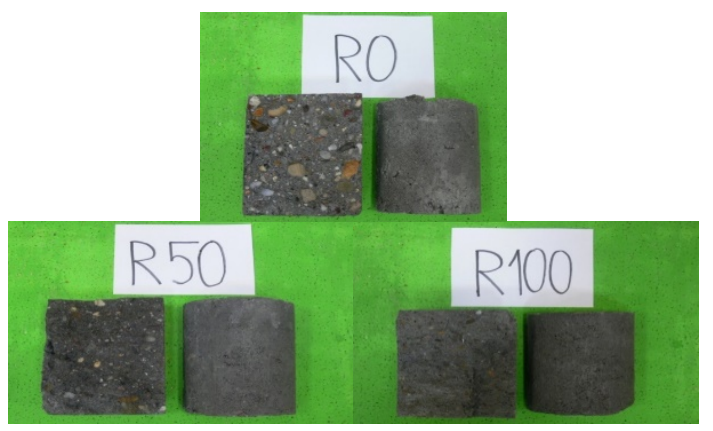

Fig. 5. Surface and fractured concrete specimens morphology. The reference sample R0, the sample with $50 \%$ substitution of natural aggregate by the RCA and the sample with $100 \%$ substitution of natural aggregate by RCA are presented. The samples with RCA appear as more "homogeneous" ones.

Table 3. Splitting tensile strength and compressive strength of shaped vibro-pressed concrete elements

\begin{tabular}{lllllll}
\multirow{2}{*}{$\begin{array}{l}\text { Sample } \\
\text { notation }\end{array}$} & \multicolumn{2}{l}{ Splitting tensile strength [MPa] } & \multicolumn{2}{l}{ Compressive strength; [MPa] } & $\begin{array}{l}\text { Densification } \\
\text { degree }\end{array}$ & w/c \\
\cline { 2 - 5 } & After 7 days & After 28 days & After 7 days & $\begin{array}{l}\text { After } \\
\text { days }\end{array}$ & 28 & \\
\hline R0 & $3,20 \pm 0,40$ & $3,85 \pm 0,20 *$ & $33,0 \pm 1,5$ & $35,0 \pm 7,5$ & 1,48 & 0,36 \\
R20 & $3,05 \pm 0,15$ & $3,60 \pm 0,30$ & $27,0 \pm 0,5$ & $34,0 \pm 5,0$ & 1,44 & 0,38 \\
R50 & $2,50 \pm 0,30$ & $2,65 \pm 0,60$ & $21,5 \pm 4,5$ & $19,0 \pm 6,0$ & 1,40 & 0,40 \\
R100 & $1,90 \pm 0,50$ & $2,45 \pm 0,47$ & $19,0 \pm 4,0$ & $29,0 \pm 6,5$ & 1,44 & 0,55 \\
Rc25 & $3,60 \pm 0,05$ & $3,90 \pm 0,20$ & $39,0 \pm 1,0$ & $40,5 \pm 2,0$ & 1,45 & 0,35 \\
Rc50 & $3,10 \pm 0,20$ & $3,55 \pm 0,25$ & $33,5 \pm 4,5$ & $39,5 \pm 4,0$ & 1,43 & 0,35 \\
Rc100 & $3,55 \pm 0,75$ & $3,10 \pm 0,30$ & $30,0 \pm 0,5$ & $30,5 \pm 3,5$ & 1,42 & 0,36 \\
Rf25 & $3,15 \pm 0,20$ & $3,70 \pm 0,30$ & $31,0 \pm 4,0$ & $41,0 \pm 1,5$ & 1,48 & 0,33 \\
Rf50 & $3,50 \pm 0,60$ & $3,90 \pm 0,15$ & $38,0 \pm 1,0$ & $40,0 \pm 0,5$ & 1,51 & 0,36 \\
Rf100 & $3,60 \pm 0,00$ & $3,75 \pm 0,35$ & $36,0 \pm 2,0$ & $34,0 \pm 4,0$ & 1,48 & 0,40 \\
\hline
\end{tabular}

*The results meeting the standard requirements are indicated

2) The Rc25 and Rf50 concretes show even slightly higher strength than the reference concrete. This is the consequence of lower w/c ratio for Rc25 mixture and better compactness of Rf50 material. One cannot exclude that the recycled material is more "flexible" and susceptible for the compaction and vibropressing and the denser microstructure can be thus formed.

3) The most significant decrease of compressive strength is observed in the case of concrete with raw, not fractioned recycled concrete aggregate. The lowest compressive strength - 55\% of the value for reference - was found for the R50 material. This effect is surprising, however such a high percentage of RCA substituting natural aggregate should not be used in practice because of too low splitting strength.

4) The reference R0 concrete shows high splitting tensile strength but relatively lower compressive strength, as compared to the concretes with substitution of sand fraction by 0/4 RCA.

5) The compressive strength of concrete with recycled concrete aggregate is in case of Rf25 material 17\% higher than that for reference R0 but a slight decrease is observed with higher RCA content. The mixture Rf100 is an exception; the strength decrease below the value for reference concrete is observed. This is because of the necessity to augment the w/c ratio.

6) The vibro-pressed concrete produced with substitution of natural aggregate by recycled concrete aggregate 0/16, with no-fractioning (R20, R50, R100) and 4/16 coarser fraction substitution (Rc25, Rc50, Rc100) reveal lower than required (3,60 $\mathrm{MPa}$ ) standard splitting tensile strength of concrete with higher aggregate content. The loss of strength results from the higher w/c and weaker mixture compaction. 


\subsubsection{Absorption capacity}

The absorption capacity was determined according to the EN 1338:2005+AC2007 standard, annex E after 28days maturing in water [19]. During 27 days the samples, formed using Econom method, attained constant mass. The average value from three individual measurements was the result in each case; the absorption capacity data are shown in fig. 6.

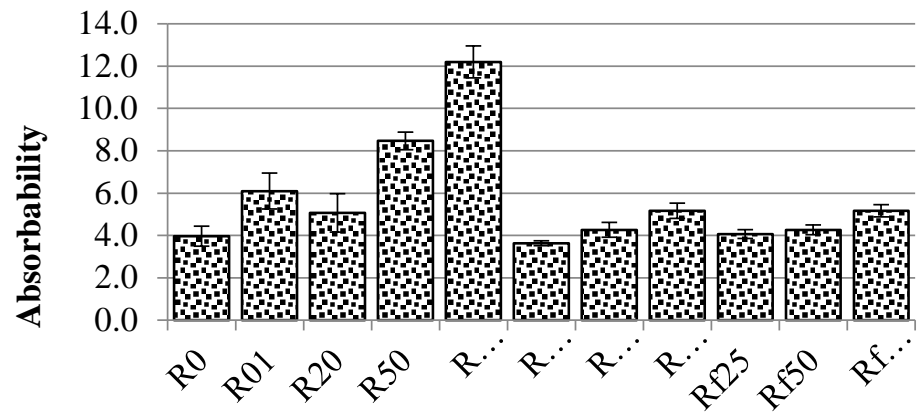

Fig. 6. Absorption capacity of vibro-pressed concretes with recycled concrete aggregate

The absorption capacity of hardened concretes is affected by the type and amount of recycled concrete aggregate. Absorption capacity becomes higher with the recycled concrete aggregate ratio. The most significant increase of absorption capacity is found for concretes with 0/16 recycled concrete aggregate. The concrete with 20, 50 and $100 \%$ substitution of natural aggregate by this RCA brings about the 27, 113 and 205\% growth of absorption capacity in respect to reference $\mathrm{R} 0$.

The substitution of natural aggregate by the $4 / 16 \mathrm{~mm}$ or $0 / 4 \mathrm{~mm}$ fractions of recycled concrete material does not result in significant absorption capacity increase. The substitution of 25\% coarse aggregate by the 4/16 RCA brings about the $10 \%$ reduction of absorption capacity. Generally, the absorption capacity of concrete elements with coarse or fine recycled concrete aggregate instead of natural sand and gravel does not exceed the 6\% limit, recommended in the EN 1338:2005+AC2007standard [19]. However one should take in mind that the durability of material with 0/16 RCA could be reduced.

The effect of w/c ratio on the absorption capacity is of special importance. In the case of reference concrete of reduced water content $(\mathrm{R} 01, \mathrm{w} / \mathrm{c}=0,27)$ the absorption capacity is $50 \%$ higher than for the reference concrete with $\mathrm{w} / \mathrm{c}=0,36$. Too low water content results in worse compaction susceptability and shortage of hydrated material coating; therefore the hydration process is disturbed and the defects of microstructure occur. As a consequence the tightness of concrete is reduced.

\section{Conclusions}

1) The recycled aggregate in the vibropressed concrete paving blocks producing plant can be produced "in situ" by grinding of damaged products and excess concrete mixture residue.

2) The vibropressed concrete elements produced with recycled concrete aggregate show fairly good mechanical properties, depending on the aggregate grain size and aggregate ratio. The best results are achieved when the 0/4 RCA fraction is introduced instead of the $0 / 2$ natural sand. As lower the substitution, as better the test results.

3) The compaction process - consistency - workability - water dosage are affected by the grain size fraction substitution of natural aggregate by the recycled concrete aggregate used. Generally, the addition of recycled concrete aggregate has no negative effect on the workability of concrete mixtures as the necessary correction of process water amount by chemical admixtures is done.

4) One should take in mind that the properties of concrete mixture and hardened material dedicated to the production of vibropressed concrete paving blocks are in some extend affected by the compaction process. For verification of applicability of materials and for feasibility studies the use of laboratory paving blocks machine and vibropressing operation should be recommended.

5) Generally, the absorption capacity of concrete elements with coarse or fine recycled concrete aggregate instead of natural sand and gravel does not exceed the standard 6\% limit. The most significant increase of absorption capacity is found for concretes with $0 / 16$ recycled concrete aggregate.

\section{References}

[1] Jamshidi A, Kurumisawa K, White G, Nishizawa T, Igarashi T, Nawa T, Mao J. State -of-the-art of interlocking concrete block pavement technology in Japan as a post-modern pavement. Con. Build. Mat. 2019; 713-755. doi.org/10.1016/j.conbuildmat.2018.11.286 
[2] Brylicki W. Concrete paving blocks produced from vibropressed concrete - handbook. ed. Polski Cement, Kraków. 1998. Access at: dockplayer.pl (in Polish).

[3] EN 13286-2: 2010 Unbound and hydraulically bound mixtures. Test methods for laboratory reference density and water content.

[4] Kadri EH, Tahar ZA, Mouret M, Rogat D. Effect of recycled aggregate on fresh concrete properties. In: Concrete recycling research and practice. edited by F. de Larrard, H. Colina. CRC Taylor \& Francis; 2019.

[5] Pellegrino C, Faleschini F. Recycled Aggregates for Concrete Production: State-of-the-Art in: Sustainability Improvement in the Concrete Industry. Use of Recycled Materials for Structural Concrete Production. Springer; 2016.

[6] Etxeberria M, Vazqez E, Mari A, Barra M. Influence of amount of recycled coarse aggregates and production process on properties of recycled aggregate concrete, Cem. Concr. Res. 2007;37(5): 735-742.

[7] Lovato PS, Possan E, Molin DCCD, Masuero AB, Ribeiro JLD, Modeling of mechanical properties and durability of recycled aggregate concretes. Con. Build. Mat. 2012; 26: 437-447. doi: 0.1016/j. conbuildmat.2011.06.043.

[8] Lotfi S, Deja J, Rem P, Mróz R, van Roekel E, van der Stelt H. Mechanical recycling of EOL concrete into high-grade aggregates. Res. Cons. Rec. 2014; 87: 117-125.

[9] Lotfi S, Rem P, Deja J, Mróz R. An experimental study on the relation between input variables and output quality of a new concrete recycling process. Con. Build. Mat. 2017:137: 128-140.

[10] Rodríguez C, Parra C, Casado G, Miñano I, Albaladejo F, Benito F, Sanchez I. The incorporation of construction and demolition wastes as recycled mixed aggregates in non-structural concrete precast pieces. J. Clean. Prod. 2016: 152-161. doi: 10.1016/j.jclepro.2016.03.137.

[11] Bao Leab Quoc-Bao Buia. Recycled aggregate concretes - A state-of-the-art from the microstructure to the structural performance. Con. Build. Mat. 2020; 257: 119522. doi.org/10.1016/j.conbuildmat.2020.119522

[12] Wang B , Yan L , Fu Q , Kasal B. A Comprehensive Review on Recycled Aggregate and Recycled Aggregate Concrete. Res. Con. Rec. 2021;171: 105565. doi.org/10.1016/j.resconrec.2021.105565

[13] Nedeljkovic M, Visser J, Savij B, Valcke S, Schlangen E. Use of fine recycled concrete aggregates in concrete. J. Build. Eng. 2021: 38:102196. doi.org/10.1016/j.jobe.2021.102196

[14] Glaydson Simões dos Reis, Quattrone M, Weslei Monteiro A, Cazacliu BG, Hoffmann CS. Current Applications of Recycled Aggregates from Construction and Demolition: A Review. Materials. 2021;14: 1700. doi.org/10.3390/ma14071700

[15] López Gayarre F, López-Colina C, Serrano MA, López-Martínez A. Manufacture of concrete kerbs and floor blocks with recycled aggregate from C\&DW. Con. Build. Mat. 2013;40:1193-1199. doi: 10.1016/j.conbuildmat.2011.11.040.

[16] Rodríguez C, Miñano I, Aguilar MA, Ortega JM, Parra C, Sánchez I. Properties of Concrete Paving Blocks and Hollow Tiles with Recycled Aggregate from Construction and Demolition Wastes. Materials. 2017; 10(12): 1374.

[17] Chu SH, Chi Sun Poon, Lam CS, Li L. Effect of natural and recycled aggregate packing on properties of concrete blocks. Con. Build. Mat. 2021: 278. doi: 16/j.conbuildmat.2021.122247

[18] EN 12620:2013 Aggregates for concrete.

[19] EN 1338:2005+AC:2007 Concrete paving blocks - Requirements and test methods.

[20] EN 15037-2:2009+A1:2011 Precast Concrete Products-Beam-and-Block Floor Systems-Part 2: Concrete Blocks.

[21] EN 12390-7:2001 Testing hardened concrete - Part 7: Density of hardened concrete.

[22] Hüsken G, Brouwers HJH. A new mix design concept for earth-moist concrete: A theoretical and experimental study. Cem. Concr. Res. 2008; 38: 1246-1259.

(C) 2021 by the author(s). This work is licensed under a Creative Commons Attribution 4.0 International License (http://creativecommons.org/licenses/by/4.0/). Authors retain copyright of their work, with first publication rights granted to Tech Reviews Ltd. 